\title{
Bimodal listeners are not sensitive to interaural time differences in unmodulated low-frequency stimuli $(L)$
}

\author{
Anneke Lenssen ${ }^{\text {a) }}$ and Tom Francart \\ Division of Experimental Otorhinolaryngology, Department of Neurosciences, Katholieke Universiteit Leuven, \\ $O \& N 2$, Herestraat 49 bus 721, B-3000 Leuven, Belgium \\ Jan Brokx \\ Department of Otorhinolaryngology and Head and Neck Surgery, University Hospital Maastricht, P.O. Box \\ 5800, 6202 AZ Maastricht, The Netherlands \\ Jan Wouters \\ Division of Experimental Otorhinolaryngology, Department of Neurosciences, Katholieke Universiteit Leuven, \\ $O \& N 2$, Herestraat 49 bus 721, B-3000 Leuven, Belgium
}

(Received 16 April 2010; revised 17 January 2011; accepted 27 January 2011)

\begin{abstract}
Sensitivity to interaural time differences (ITDs) with unmodulated low-frequency stimuli was assessed in bimodal listeners who had previously shown to be good performers in ITD experiments. Two types of stimuli were used: (1) an acoustic sinusoid combined with an electric transposed signal and (2) an acoustic sinusoid combined with an electric clicktrain. No or very low sensitivity to ITD was found for these stimuli, even though subjects were highly trained on the task and were intensively tested in multiple test sessions. In previous studies with users of a cochlear implant (CI) and a contralateral hearing aid (HA) (bimodal listeners), sensitivity was shown to ITD with modulated stimuli with frequency content between 600 and $3600 \mathrm{~Hz}$. The outcomes of the current study imply that in speech processing design for users of a $\mathrm{CI}$ in combination with a HA on the contralateral side, the emphasis should be more on providing salient envelope ITD cues than on preserving fine-timing ITD cues present in acoustic signals. (C) 2011 Acoustical Society of America.
\end{abstract}

[DOI: $10.1121 / 1.3557051]$

PACS number(s): 43.66.Qp, 43.66.Ts, 43.64.Me [JCM]

Pages: $3457-3460$

\section{INTRODUCTION}

In normal hearing $(\mathrm{NH})$ listeners, the localization of lowfrequency sounds (below $1500 \mathrm{~Hz}$ ) is mainly based on interaural time differences (ITDs) (Kuhn, 1977). Physically, for a human head, the largest available ITD is around $690 \mu \mathrm{s}$, when a sound arrives from the side of the head (Feddersen et al., 1957). A just noticeable difference (JND) in ITD down to 10 $\mu \mathrm{s}$ is found for sinusoids below $1500 \mathrm{~Hz}$ in $\mathrm{NH}$ listeners (Yost, 1974). For higher frequencies the ITD becomes ambiguous, since the period becomes smaller than physically available ITDs.

Francart et al. (2009, 2011), have shown that bimodal listeners can be sensitive to ITDs provided in the mid to basal regions of the cochlea using pulse trains of $100 \mathrm{pps}$ electrically combined with filtered clicktrains of $100 \mathrm{~Hz}$ presented acoustically. In the same subjects, sensitivity to ITD was found using transposed stimuli with carrier frequencies between 600 and $3600 \mathrm{~Hz}$. At this moment no data are available that show ITD sensitivity for bimodal listeners with unmodulated stimuli below $600 \mathrm{~Hz}$.

For bilateral cochlear implant (CI) users, ITD sensitivity for single electrode pulse trains was shown (van Hoesel and Tyler, 2003; Long et al., 2003; Majdak and Laback, 2006; Laback et al., 2007; van Hoesel, 2007). For low-frequency unmodulated pulse trains of $100 \mathrm{pps}$ and modulated high-

\footnotetext{
a) Author to whom correspondence should be addressed. Electronic mail: anneke.lenssen@med.kuleuven.be
}

frequency pulse trains with a modulation rate of $100 \mathrm{~Hz}$ performance are comparable. For higher pulse rates or modulation rates, ITD sensitivity decreased for both modulated pulse trains and unmodulated stimuli, performance was however higher for unmodulated stimuli than for modulated stimuli (van Hoesel, 2007; van Hoesel et al., 2009). In neurophysiologic studies with animals, a similar difference in responses to modulated and unmodulated acoustic stimuli was found. A decrease in neural responses to ITD with increasing modulation frequency was found for modulated stimuli, with a limit around $300 \mathrm{~Hz}$, there was no decrease in performance for the pure tone frequencies tested up to $400 \mathrm{~Hz}$ (Griffin et al., 2005).

The decrease in ITD sensitivity with increasing pulse rate in bilateral CI users was also seen in NH listeners for ITD in the envelope of modulated stimuli (Bernstein and Trahiotis, 2002) but not for pure tones, where sensitivity increases for frequencies up to $1000 \mathrm{~Hz}$ (Klumpp and Eady, 1956).

Bernstein and Trahiotis (2002) used a so-called transposed stimulus, a high rate sinusoidal carrier modulated with a half wave rectified sinusoid, to provide the high-frequency auditory channels with a similar neural activation pattern as a low-frequency signal in the low-frequency channels. They found that for a high-frequency carrier, ITD detection performance with transposed tones was better than for sinusoidal amplitude modulated tones, and even comparable to performance with pure tones for modulation frequencies below $128 \mathrm{~Hz}$. Laback et al. (2009), Ewert et al. (2010), and Bernstein and Trahiotis (2009) studied the influence of different temporal aspects of the envelope on ITD detection 
performance. Bernstein and Trahiotis (2009) used a raised-sine stimulus with varying exponent and found a positive effect on ITD discriminability of increasing sharpness of the envelope. Also Laback et al. (2009) and Ewert et al. (2010) found a decrease in threshold ITD with increasing slope steepness in NH listeners using high-frequency stimuli with a low-frequency trapezoidal modulator. This effect was not found in bilateral CI users. In these studies also an increasing off-time between the peaks was found to improve the detection of ongoing ITD for both NH listeners and CI users.

In the current study, sensitivity of bimodal listeners to ITDs in low-frequency stimuli was assessed. Since in $\mathrm{NH}$ and in bilateral CI listeners, ITD detection performance was higher for unmodulated than for modulated stimuli, and bimodal listeners generally have access to low-frequency residual hearing in the ear contralateral to their $\mathrm{CI}$, they could show sensitivity to ITDs presented in this frequency region.

Two stimuli types were used. In the first stimulus the electric signal was a transposed sinusoid, with a high rate carrier and a low-frequency envelope. In normal hearing, this stimulus results in a similar activation pattern as an acoustic sinusoid passed through cochlear filtering according to the model proposed in Bernstein and Trahiotis (2002). In the second stimulus the electric signal was a low-frequency pulse train, of the same frequency as the acoustic signal. Compared to the transposed sinusoidal stimulus, this stimulus has a large off-time and a steep onset within each stimulus period, which is according to Laback et al. (2009) and Ewert et al. (2010), expected to increase ITD sensitivity. The acoustic signal was a low-frequency sinusoid with frequencies between 100 and $400 \mathrm{~Hz}$ in both experiments.

\section{METHODS}

\section{A. Subjects}

Three bimodal listeners were selected from a set of subjects who participated in previous experiments. They received their CI at the University Hospital (UZ) Leuven or the Academic Hospital Maastricht (AZM). These subjects were selected based on their good sensitivity to envelope ITDs in previous tests (Francart et al., 2009, 2011). They were volunteers and signed an informed consent form. They wore a hearing aid (HA) contralaterally to their CI on a daily basis. S02 and S11 had an electrode array of the contour type, S12 had an array of the contour advance type. They all used a Freedom processor. More detailed information on each of the subjects and audiograms of the acoustically stimulated ear of each subject can be found in Francart et al., (2011).

\section{B. Setup}

All stimuli were presented under direct computer control, not using the subjects own external devices. Stimuli were presented using the APEX 3 program developed at ExpORL (K. U. Leuven) (Francart et al., 2008). For acoustic stimulation a RME Multiface II DSP sound card (RME, Germany) was used, connected to a single insert phone of type Etymotic ER-3A. The insert phone was calibrated using a $2 \mathrm{cc}$ coupler to conform the ISO389 norm. For electric stimulation the NICv2interface was used in combination with a L34 experimental processor provided by Cochlear Ltd. With the sound card a trigger pulse was sent to the L34 to ensure synchronous stimulation. The relative timing of the acoustic and electric signals was checked using the output of an implant-in-a-box and the electrical output of the sound card visualized on an oscilloscope. The synchronization was done such that the first edge of the electric pulse coincided with the top of the first peak of an acoustic click.

\section{Stimuli}

Two different stimulus types were used. A stimulus consists of an acoustic signal presented to the ear contralateral to the CI, and an electric signal which is sent to the internal part of the CI. In both stimuli the acoustic signal was a sinusoid. In the first stimulus type the electric signal was a transposed sinusoid with a carrier of $6300 \mathrm{pps}$ and a modulation rate equal to the frequency of the acoustic signal. The frequencies of the acoustic signals and the accompanying modulation rates of the electric signal were 100,150, 200, 250, 280, 300, and 400 $\mathrm{Hz}$. In the second stimulus type the electric signal was a pulse train with a rate matched to the frequency of the acoustic signal. The same frequencies were tested as with the first stimulus. Cosinusoidal ramping of $50 \mathrm{~ms}$ was applied to all signals to reduce the use of onset or offset cues for ITD detection. The length of the stimuli, including onset and offset ramps, was $1 \mathrm{~s}$. For the electric signal in all stimuli the most apical electrode was used for stimulation. For S11 and S12 this was electrode 1, for S02 this was electrode 3, since the first two electrodes did not yield a clear percept. Electrodes are numbered from apex (1) to base (22). A graphical representation of the two stimulus types is shown in Fig. 1. All electric pulses were presented in monopolar mode $(\mathrm{MP} 1+2)$, with a bipolar pulse shape. The phase width was $25 \mu$ s and the inter phase gap was $8 \mu$ s.

\section{Procedure}

The same procedure was used as in previous studies assessing ITD sensitivity in bimodal subjects (Francart et al., 2009, 2011). This balancing procedure is based on extent of lateralization and consists of three steps. First, the loudness of the acoustic and the electric signals were separately set to
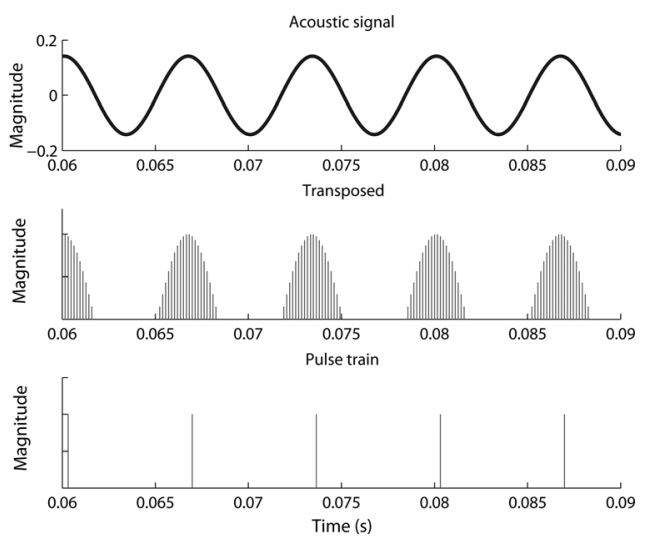

FIG. 1. Segment of an example acoustic signal and of the two electrical signal types. Top: the acoustic sinusoid with a frequency of $150 \mathrm{~Hz}$, middle: electric transposed signal with a carrier rate of $6300 \mathrm{pps}$ and a modulation rate of $150 \mathrm{pps}$, bottom: electric pulse train with a rate of $150 \mathrm{pps}$. 
a comfortable level. Secondly, the signals were balanced in loudness. In the third step the resulting loudness balance was varied and adjusted by aiming to shift the stimulus equally far to both sides of the head.

In the first step, the acoustic and electric signals were played separately and the subjects had to rate the level of each signal as "good" on a seven point scale ranging from "inaudible" to "uncomfortably loud." In the second step, the signals were played simultaneously in both ears and the loudness balance was adjusted by changing the level of the electric signal until the stimulus sounded equally loud in both ears. Mostly the loudness of the electric signal was adjusted, except for when the subject preferred the loudness of the acoustic signal to be adjusted, this was the case for some conditions with subject S02. The exact ITD at which the ears are stimulated synchronously is not known. Francart et al. (2009) found a value of $1.5 \mathrm{~ms}$ to result in synchronous stimulation between electric and acoustic signals when using clicktrain stimuli. To minimize the influence of large asynchrony between the acoustic and electric signal on the loudness balance, as a starting value, the electric signal was delayed by $1.5 \mathrm{~ms}$ when balancing the loudness. In the final step, the loudness balance was refined by adjusting this delay through the assessment of the extent of lateralization. It was attempted to shift the stimulus to either side of the head by introducing large ITDs, ranging from 0.5 up to $2.5 \mathrm{~ms}$ in both directions. Each stimulus was presented to the subject multiple times, and the subject was asked to indicate whether it was perceived shifted to the left or to the right, with respect to the center of their head. In this step, two parameters are varied depending on the response of the subject. If the stimulus could not be shifted to both sides of the head equally far, the loudness of the electric signal was slightly adjusted and again the extent of lateralization was assessed. If the stimulus could be shifted to both sides equally far but not symmetrical around the current delay, a new estimate of the delay was made, followed by extensive assessment of the extent of lateralization. This step was iteratively performed to result in optimal delay and loudness balance. When the stimulus could consistently be shifted equally far to both sides of the head, the stimulus was considered balanced in level and time.

When the subject was able to lateralize the stimuli containing ITDs during the training, the JND in ITD was determined. To obtain this, a psychometric function for ITD was estimated using a single interval, two alternatives forced choice constant stimuli procedure. In a single run, a number of ITDs was selected over a certain range around the perceptual center and a stimulus containing each ITD was presented three times. The subject had to indicate whether the sound was lateralized to the left or to the right side. The ITDs to be presented in a single run were selected by the experimenter based on the previous subject performance. Psychometric functions were determined using the same method as in Francart et al. $(2009,2011)$. Multiple runs of the same condition (stimulus type) were done during one test session, to make sure a JND in ITD was minimally based on 30 trials per condition. From each psychometric function, the JND in ITD was determined from the slope at 50\% correct as the change in ITD necessary for a $25 \%$ change in performance. Each subject was tested in 4-5 test sessions of approximately $2 \mathrm{~h}$.
TABLE I. JND in ITD per subject for the electrical transposed signal (T) and for the stimulus containing the pulse train $(\mathrm{P})$. Stimuli frequencies are given in hertz, the JND in ITD in microsecond. No sensitivity to ITD was indicated with a "x." If the stimulus was not tested, it was kept empty. Data from previous studies were added showing subjects best and worst performance measured using a filtered clicktrain stimulus (Francart et al., 2009).

\begin{tabular}{|c|c|c|c|c|c|c|c|c|c|c|c|c|c|c|c|c|}
\hline \multirow[b]{2}{*}{ Subject } & \multicolumn{2}{|c|}{100} & \multicolumn{2}{|c|}{150} & \multicolumn{2}{|c|}{200} & \multicolumn{2}{|c|}{250} & \multicolumn{2}{|l|}{280} & \multicolumn{2}{|c|}{300} & \multicolumn{2}{|c|}{400} & \multirow[b]{2}{*}{ Best } & \multirow[b]{2}{*}{ Worst } \\
\hline & $\mathrm{T}$ & $\mathrm{P}$ & $\mathrm{T}$ & $\mathrm{P}$ & $\mathrm{T}$ & $\mathrm{P}$ & $\mathrm{T}$ & $\mathrm{P}$ & $\mathrm{T}$ & $\mathrm{P}$ & $\mathrm{T}$ & $\mathrm{P}$ & $\mathrm{T}$ & $\mathrm{P}$ & & \\
\hline $\mathrm{S} 02$ & $\mathrm{X}$ & & $\mathrm{x}$ & $\mathrm{X}$ & $\mathrm{X}$ & $\mathrm{X}$ & $\mathrm{x}$ & $\mathrm{x}$ & $\mathrm{X}$ & & $\mathrm{x}$ & $\mathrm{x}$ & $\mathrm{x}$ & $\mathrm{X}$ & 156 & 713 \\
\hline S11 & & $\mathrm{X}$ & $\mathrm{x}$ & $\mathrm{x}$ & 855 & $\mathrm{X}$ & $\mathrm{x}$ & $\mathrm{x}$ & & $\mathrm{x}$ & $\mathrm{x}$ & $\mathrm{x}$ & & & 254 & 2322 \\
\hline S12 & 590 & 636 & $\mathrm{X}$ & $\mathrm{X}$ & $\mathrm{X}$ & $\mathrm{X}$ & $\mathrm{x}$ & $\mathrm{x}$ & 1284 & $\mathrm{x}$ & $\mathrm{x}$ & $\mathrm{x}$ & & & 91 & 310 \\
\hline
\end{tabular}

\section{RESULTS}

The JND in ITD of the stimuli that were tested for each subject are shown in Table I, the stimuli for which no sensitivity to ITD was found were marked with a $\mathrm{x}$, the conditions that were not tested are kept empty.

There was little difference in performance between the two stimulus types. Generally, none of the subjects showed good sensitivity to ITDs for the stimuli that were used. They all reported that the task was very difficult and the sound was perceived as diffuse. Subject S02 reported for most stimuli after loudness balancing that the acoustic and electric signals were perceived as separate images. He was not able to lateralize any of the stimuli with the use of ITD. For subject S11, the sound image was fused and for frequencies between 200 and $300 \mathrm{~Hz}$, for large ITDs (of $1.5 \mathrm{~ms}$ ) he reported a small shift in the image location, but could not perform the lateralization task. For the $200 \mathrm{~Hz}$ stimulus, a JND in ITD was found of $855 \mu \mathrm{s}$. Subject S12 had a minimal JND in ITD of $590 \mu \mathrm{s}$ for the $100 \mathrm{~Hz}$ stimulus with a transposed electrical signal and a JND of $636 \mu$ s for the pulse train. He reported the task to be extremely difficult and tiring. None of the other stimuli could be lateralized based on ITD.

\section{DISCUSSION}

The results show no or very low sensitivity to ITDs, for the three subjects participating in the tests, with both the transposed stimulus and the pulse trains.

Although the number of subjects participating in the study was limited to three, they were good performers in previous ITD tests (Francart et al., 2009, 2011). They participated in the tests of ITD sensitivity for a minimum of $40 \mathrm{~h}$ over the year prior to this study, so they were all highly trained on the task.

From the psychometric curve, besides the JND in ITD, also an estimate of the delay of the electric signal needed for the electric and acoustic signals to be received synchronously at the auditory nerve could be extracted by determining the ITD at $50 \%$ correct. The delays obtained in the current study ranged between 0.8 and $2.2 \mathrm{~ms}$ and a slight trend could be observed of an increasing delay with a decrease in stimulus frequency. However due to the limited number of data points and the limited number of trials, the data do not provide strong statistical evidence.

As was recently shown by Laback et al. (2009) and Ewert et al. (2010), slope steepness and off-time influence 
the detection of ITD. Adjusting the stimulus waveform by increasing the off-time between the peaks and slope steepness by using the pulse train signal electrically, did not have an effect since no differences were found in performance between the two stimuli used in this study.

In a previous study on ITD sensitivity in bimodal listeners, a limitation to the modulation rate at which ITDs could be detected for transposed tones was found at $200 \mathrm{~Hz}$ (Francart et al., 2011). This finding was similar to the limit in sensitivity to the envelope ITD found in NH subjects by Bernstein and Trahiotis (2002) using transposed stimuli. A comparable rate limitation was found in neurophysiological studies with animals (Griffin et al., 2005; Snyder et al., 1995). A similar rate limitation could explain the absence of ITD sensitivity found here for the highest frequencies that were tested, but not for all stimuli since also stimuli rates of 100 and $150 \mathrm{~Hz}$ were used which are below the rate limit discussed above.

In the current study, electrically the place of stimulation in the cochlea was kept constant at the most apical electrode. Acoustically the frequency of the sinusoid was varied resulting in a change in place of stimulation in the cochlea. No change in performance was found with varying the place of stimulation acoustically. A mismatch in the place of stimulation in the cochlea between the ears can reduce sensitivity to the ITDs in the stimuli presented to the listeners (Nuetzel and Hafter, 1981). In measurements using computer tomography (CT) scans by Ketten et al. (1998), the characteristic frequency $(\mathrm{CF})$ of the most apical electrode was estimated between 387 and $2596 \mathrm{~Hz}$ in 20 nucleus CI recipients. In Boex et al. (2006) measurements of the frequency-position function resulted in estimates varying between 100 and $570 \mathrm{~Hz}$ for the most apical electrode. Inter-subject variabilities were large and the reported CFs are mostly rather high compared to the low-frequency stimuli used in the current study, meaning the most apical electrode may not have reached to the place matching the acoustically stimulated region.

In a previous study with bimodal listeners, subjects were sensitive to ITD presented in transposed stimuli with carrier frequencies between 600 and $6300 \mathrm{~Hz}$ at a middle electrode. Here, unmodulated low-frequency stimuli were used at an apical electrode. These stimuli have a narrower frequency response than the transposed stimuli used previously. A mismatch in the place of stimulation might therefore have a larger influence on sensitivity to ITD. This effect combined with a limit in neural sensitivity to ITD at higher rates could have caused the lack of sensitivity to ITD found here.

In summary, the subjects showed no or very low sensitivity to ITD for the low-frequency stimuli that were used in the current study. The same subjects previously showed sensitivity to ITD in the envelope of modulated stimuli. These findings indicate that users of a CI in combination with a contralateral HA may benefit more from a sound processing strategy providing salient envelope cues rather than fine-timing cues from the acoustic sound for the detection of ITDs.

\section{ACKNOWLEDGMENTS}

This study was supported by the IWT-Vlaanderen and Cochlear Project No. 080304. Author A.L. was supported by the EU-ITN Project AUDIS, and author T.F. was supported with a Post Doctoral Mandate (BOF) from the K.U. Leuven. We thank our subjects who voluntarily spent many hours enthusiastically participating in our experiments, and the staff of the Audiology Department of the Hospital of Maastricht and Hospital of Leuven for their cooperation.

Bernstein, L., and Trahiotis, C. (2002). "Enhancing sensitivity to interaural delays at high frequencies by using transposed stimuli," J. Acoust. Soc. Am. 112, 1026-1036.

Bernstein, L., and Trahiotis, C. (2009). "How sensitivity to ongoing interaural temporal disparities is affected by manipulations of temporal features of the envelopes of high-frequency stimuli," J. Acoust. Soc. Am. 125, 3234-3242.

Boex, C., Baud, G., Cosendai, G., Sigrist, A., Kos, M., and Pelizzone, M. (2006). "Acoustic to electric pitch comparisons in cochlear implant subjects with residual hearing," J. Assoc. Res. Otolaryngol. 7, 110-124.

Ewert, S., Dietz, M., Klein-Henning, M., and Hohmann, V. (2010). The Neurophysiological Bases of Auditory Perception; The Role of Envelope Wave Form, Adaptation, and Attacks in Binaural Perception (Springer, New York), Chap. 32, pp. 337-346.

Feddersen, W., Sandel, T., Teas, D., and Jeffress, L. (1957). "Localization of high-frequency tones," J. Acoust. Soc. Am. 29, 988-991.

Francart, T., Brokx, J., and Wouters, J. (2009). "Sensitivity to interaural time differences with combined cochlear implant and acoustic stimulation," J. Assoc. Res. Otolaryngol. 10, 131-141.

Francart, T., Lenssen, A., and Wouters, J. (2011). "Sensitivity of bimodal listeners to interaural time differences with modulated single- and multiple-channel stimuli," Audiol. Neuro- Otol. 16, 82-92.

Francart, T., van Wieringen, A., and Wouters, J. (2008). "Apex3: A multipurpose test platform for auditory psychophysical experiments," J. Neurosci. Methods 172, 283-293.

Griffin, S., Bernstein, L., Ingham, N., and McAlphine, D. (2005). "Neural sensitivity to interaural envelope delays in the inferior colliculus of the guinea pig," J. Neurophysiol. 93, 3463-3478.

Ketten, D., Skinner, M., Wang, G., Vannier, M., Gates, G., and Neely, J. (1998). "In vivo measures of cochlear length and insertion depth of nucleus cochlear implant electrode arrays," Ann. Otol. Rhinol. Laryngol. 107, 1-16.

Klumpp, R., and Eady, H. (1956). "Some measurements of interaural time difference thresholds," J. Acoust. Soc. Am. 28, 859-860.

Kuhn, G. (1977). "Model for the interaural time differences in the azimuthal plane," J. Acoust. Soc. Am. 62, 157-167.

Laback, B., Majdak, P., and Baumgartner, W. (2007). "Lateralization discrimination of interaural time delays in four-pulse sequences in electric and acoustic hearing," J. Acoust. Soc. Am. 121, 2182-2191.

Laback, B., Zimmerman, I., and Majdak, P. (2009). "Perception of interaural time differences in electric and acoustic hearing," in Proceedings of the 2nd International Symposium on Auditory and Audiological Research (ISAAR), Elsinore, Denmark, pp. 403-416.

Long, C., Eddington, D., Colburn, H., and Rabinowitz, W. (2003). "Binaural sensitivity as a function of interaural electrode position with a bilateral cochlear implant user," J. Acoust. Soc. Am. 114, 1565-1574.

Majdak, P., and Laback, B. (2006). "Effects of interaural time difference in fine structure and envelope of lateral discrimination in electric hearing," J. Acoust. Soc. Am. 120, 2190-2201.

Nuetzel, J., and Hafter, E. (1981). "Lateralization of complex waveforms: Spectral effects," J. Acoust. Soc. Am. 69, 1112-1118.

Snyder, R., Leake, P., Rebscher, S., and Beitel, R. (1995). "Temporal resolution of neurons in cat inferior colliculus to inbracochlear electrical stimulation: Effects of neonatal deafening and chronic stimulation," J. Neurophysiol. 73, 449-467.

van Hoesel, R. (2007). "Sensitivity to binaural timing in bilateral cochlear implant users," J. Acoust. Soc. Am. 121, 2192-2206.

van Hoesel, R., Jones, G., and Litovsky, R. (2009). "Interaural time-delay sensitivity in bilateral cochlear implant users: Effect of pulse rate, modulation rate and place of stimulation," J. Assoc. Res. Otolaryngol. 10, $557-567$.

van Hoesel, R., and Tyler, R. (2003). "Speech perception, localization and lateralization with bilateral cochlear implants," J. Acoust. Soc. Am. 113, 1617-1630.

Yost, W. (1974). "Discrimination of interaural phase differences," J. Acoust. Soc. Am. 55, 1299-1303. 